\title{
Discovery in Hydrating Plaster Using Machine Learning Methods
}

\author{
Judith E. Devaney and John G. Hagedorn \\ National Institute of Standards and Technology, Gaithersburg MD, 20899-8951, USA, \\ \{judith.devaney, john.hagedorn\}@nist.gov, \\ http://math.nist.gov/mcsd/savg
}

\begin{abstract}
We apply multiple machine learning methods to obtain concise rules that are highly predictive of scientifically meaningful classes in hydrating plaster over multiple time periods. We use three dimensional data obtained through X-ray microtomography at greater than one micron resolution per voxel at five times in the hydration process: powder, after 4 hours, 7 hours, 15.5 hours, and after 6 days of hydration. Using statistics based on locality, we create vectors containing eight attributes for subsets of size $100^{3}$ of the data and use the autoclass unsupervised classification system to label the attribute vectors into three separate classes. Following this, we use the C5 decision tree software to separate the three classes into two parts: class 0 and 1, and class 0 and 2 . We use our locally developed procedural genetic programming system, GPP, to create simple rules for these. The resulting collection of simple rules are tested on a separate $100^{3}$ subset of the plaster datasets that had been labeled with their autoclass predictions. The rules were found to have both high sensitivity and high positive predictive value.

The classes accurately identify important structural comonents in the hydrating plaster. Morover, the rules identify the center of the local distribution as a critical factor in separating the classes.
\end{abstract}

\section{Introduction}

Plaster of paris is a widely used material of economic importance [1. For example, the porcelain industry maintains large numbers of molds whose strength, durability, and ability to absorb water impact the industry's costs [2].

Plaster powder is formed by calcining gypsum (calcium sulfate dihydrate, $\left.\mathrm{CaSO}_{4} \cdot 2 \mathrm{H}_{2} \mathrm{O}\right)$ to form calcium sulfate hemihydrate $\left(\mathrm{CaSO}_{4} \cdot \frac{1}{2} \mathrm{H}_{2} \mathrm{O}\right)$. The solid plaster is then formed by adding water (hydration) to the powder and allowing the mixture to set. The equations are [1]:

Calcination: $\mathrm{CaSO}_{4} \cdot 2 \mathrm{H}_{2} \mathrm{O}=\mathrm{CaSO}_{4} \cdot \frac{1}{2} \mathrm{H}_{2} \mathrm{O}+\frac{3}{2} \mathrm{H}_{2} \mathrm{O}$

Hydration: $\mathrm{CaSO}_{4} \cdot \frac{1}{2} \mathrm{H}_{2} \mathrm{O}+\frac{3}{2} \mathrm{H}_{2} \mathrm{O}=\mathrm{CaSO}_{4} \cdot 2 \mathrm{H}_{2} \mathrm{O}$

During hydration, an interlocking network of gypsum crystals forms. See Figure 1 for a scanning electron micrograph (900X) [3] of precipitated gypsum crystals $\left(\mathrm{CaSO}_{4} \cdot 2 \mathrm{H}_{2} \mathrm{O}\right)$. This crystalline network is the foundation of the strength, 
durability, and absorptivity of the plaster 1]. However, the final properties of the set plaster are dependent on many things such as the water-solid ratio in hydration, impurities in the plaster, additives, temperature, and production conditions [3]. Moreover, these interact. For example, as the water-solid ratio in the hydrating plaster increases, the volume fraction of porosity increases, absorptivity of the plaster increases, but the strength and durability decrease [1. There is much to learn about plaster; even the form of the kinetic equations (fraction of plaster reacted versus time) is not agreed upon [4] [5] 6]. Understanding the process of setting plaster as well as being able to predict its final properties is of scientific as well as economic interest.

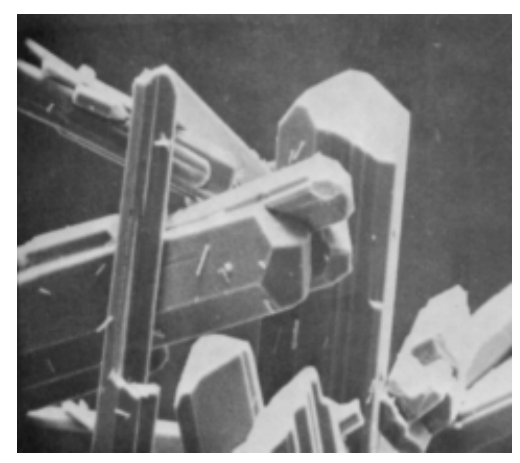

Fig. 1. A scanning electron micrograph (900X) 3] of precipitated gypsum crystals $\left(\mathrm{CaSO}_{4} \cdot 2 \mathrm{H}_{2} \mathrm{O}\right)$.

\section{High Resolution Data}

Recently, X-ray microtomography has been used to obtain the unprecedented resolution of $0.95 \mu \mathrm{m}$ per voxel in three dimensional images of hydrating plaster. Commercial grade plaster of paris was mixed with a water-to-solids mass ratio of 1.0 and viewed with X-ray microtomography after 4, 7, 15.5 hours and 6 days. Additionally, a separate sample of plaster powder was imaged. This resulted in five images of plaster of size $1024^{3}$. This is gray scale data with each voxel varying from 0 to 255 [7] 8 .

\section{Methodology}

We seek simple rules to describe and predict structural components in hydrating plaster such as hydration products (gypsum crystals), unhydrated plaster, and porosity (holes). With materials like cement, it is straightforward to obtain such components through thresholding of the brightness histogram. However, this is 
not the case with plaster [8]. Since the problem is not straightforward, we use multiple methods from machine learning to obtain the rules. We use a combination of unsupervised classification, decision trees, and genetic programming to obtain these rules. The rules are developed on a $100^{3}$ subset of the data taken at the same place in each dataset. The rules are tested on a completely separate $100^{3}$ subset of the data taken at the same place in each dataset.

\subsection{Unsupervised Classification}

Since the data is unlabeled, we start the discovery process with an unsupervised classifier. We use autoclass [9] 10, 11, 12] 13] which has been used successfully for discovery. In the creation of attributes for input to autoclass, we follow the Principle of locality [14, wherein natural laws are viewed as the consequence of small-scale regularities. Since the particle sizes may be as small as a few microns [8], we choose as our scale a $3^{3}$ cube centered on each pixel in the image. Using simple statistics on these small cubes, we create eight attributes for each pixel as input vectors to autoclass, as described in the following table.

\begin{tabular}{cl} 
Name & \multicolumn{1}{c}{ Definition } \\
\hline A0 & gray level value of pixel itself \\
A1 & neighborhood midrange \\
A2 & neighborhood variance about midrange \\
A3 & neighborhood range \\
A4 & neighborhood minimum \\
A5 & neighborhood maximum \\
A6 & neighborhood median \\
A7 & neighborhood mean
\end{tabular}

Hence, for the $100^{3}$ training subcube, this results in 1,000, 000 vectors. Because materials scientists are interested in three classes within hydrating plaster [15, we constrain autoclass to seek three classes. Classification runs were performed for each training subcube for powder, 4 hours, 7 hours, 15.5 hours, and 6 days. Since this data is at a new resolution, we do not have pre-labeled data to compare it with, or experts who can label it.

We validate the classification through a visual comparison with the classes obtained. The $100^{3}$ dataset used for training is small enough to look at all one hundred $100^{2}$ images in the dataset and in the classification. A hundred $100^{2}$ images can be printed on an $8 \frac{1}{2}$ by 11 page in a ten by ten array making comparisons straightforward. Due to space considerations, we reproduce three images from each array here. Each image is taken at $\mathbf{z}=0,30,60$ in the image array. Figure 2 shows the data and classes for the plaster powder. The data is on the left, with $\mathbf{z}=0$ at the bottom, $\mathbf{z}=30$ in the middle, and $\mathbf{z}=60$ at the top. The corresponding classification for each plane is to the right of the data. Class 0 is black, class 1 is gray, and class 2 is white. 


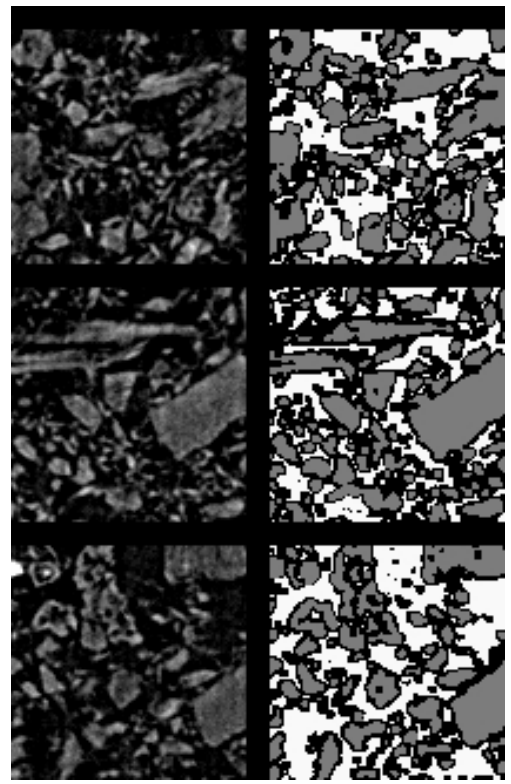

Fig. 2. Comparison of three $100^{2}$ slices of original data with corresponding classification for powder. The data is in the left column, with $\mathbf{z}=0$ at the bottom, $\mathbf{z}=30$ in the middle, and $\mathbf{z}=60$ at the top. The corresponding classification for each plane is to the right of the data.

It is immediately obvious that autoclass has picked up the basic structure of the data. The plaster particles are in class 1. Figure 3 shows the equivalent images for 4, 7, 15,5 hours and 6 days of hydration. Again the structure in the data matches the structure of the classification. In the classified data, Class 2 (the white area) is the pore space, Class 1 (the grey area) identifies the crystalline network and the unhydrated plaster, and Class 0 (the black area) represents the boundary region. Figure 4 shows this difference clearly in renderings of the individual classes in their three dimensional configuration. Plaster hardens with little change in external volume, but since the volume of the hydration products is smaller than the original material plus water, voids occur inside [16]. In our classification, the space seems to form around the gypsum crystals.

\subsection{Decision Tree}

In order to gain better insight into the classifications for each time period, we seek comprehensible representations of the classification algorithms. That is, we wanted to find relatively simple rules to determine each element's class based on its eight attributes. But autoclass operates in a "black-box" fashion. The algorithm by which it classifies and predicts elements is opaque to the user. 

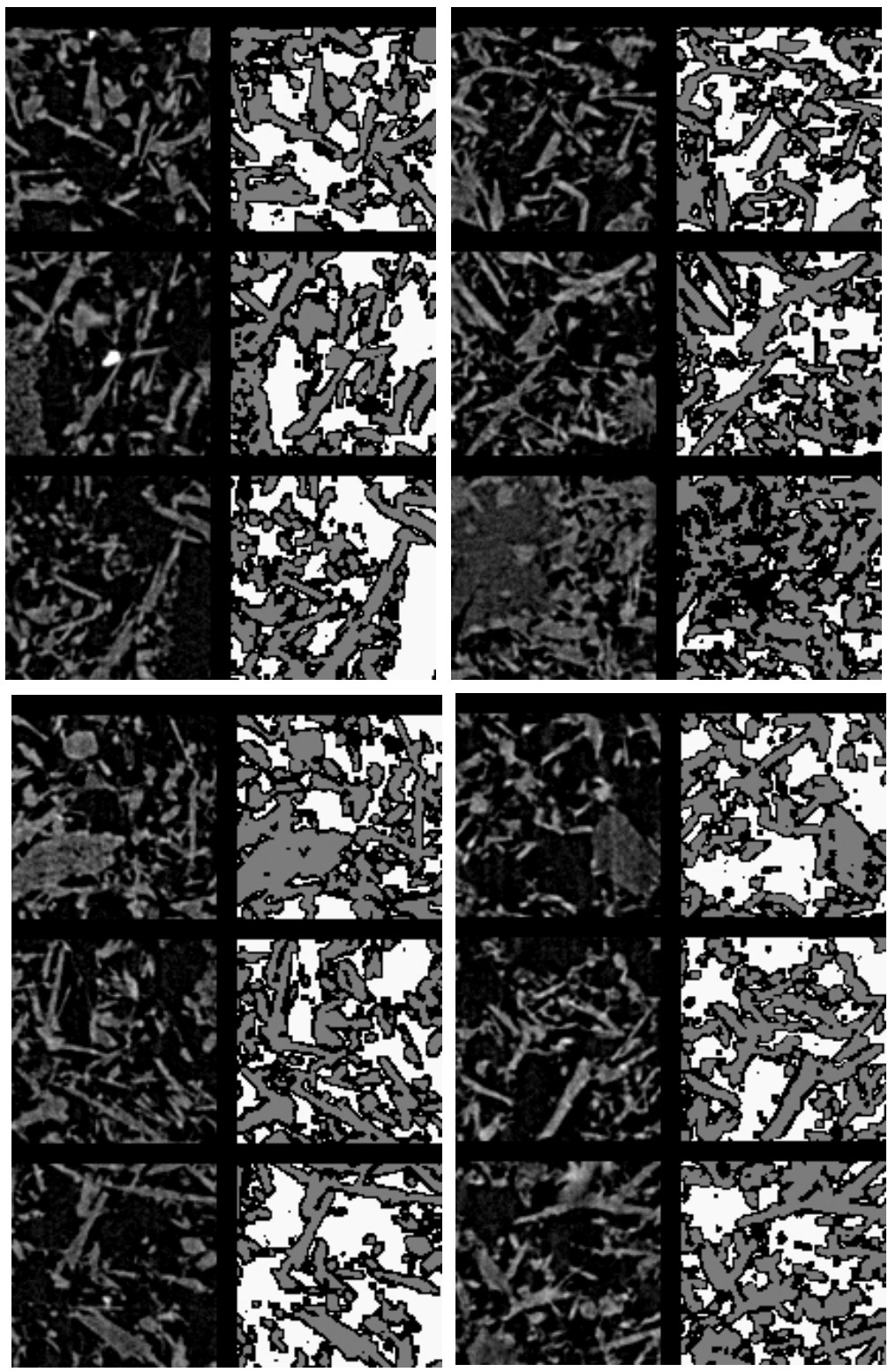

Fig. 3. Comparisons of original data with classification for 4 hours (top left), 7 hours (top right), 15.5 hours (bottom left), and 6 days (bottom right). For each time, three $100^{2}$ slices of original data are in the left column, with $\mathbf{z}=0$ at the bottom, $\mathbf{z}=30$ in the middle, and $\mathbf{z}=60$ at the top. The corresponding classification for each plane is to the right of the data. 
To derive more transparent statements of the classification schemes, we used a decision tree, C5 17]. C5 is the commercial successor to C4.5 18, which has been used extensively for learning.

Runs of C5 on the autoclass labeled attributes produced incomprehensible trees with thousands of nodes. However, the component classes in the brightness histograms in Figure 5 indicated that class 2 and class 1 were easily separable. Ten fold cross validation on the combined class 1 and 2 showed that this was the case in four of the five datasets. Three of the datasets yielded single node decision stumps with less than five missclassifications over hundreds of thousands of cases for powder, 4 hours, and 15.5 hours. All of these branched on attribute A1. The fourth simple classification was for the 7 hour dataset. This also yielded a single node decision stump; however, this branched on A7. For uniformity in the final rules across the hydration times, the 7 hour case was rerun requiring C5 to use only A1 to get a single best split on this attribute. The six day dataset did not yield a simple decision tree for the combined class 1 and 2. So it was also run with A1 as the only attribute to get the best split for input into the next phase, which was genetic programming to obtain complete and simple rules.

The attribute A1 is the local midrange. The midrange is a robust estimator of the center of a short tailed distribution [19]. Since the range is limited for each neighborhood to $0-255$, this is the situation here. So all the rules are now of the form: if the center of the local distribution is $\leq x \ldots$
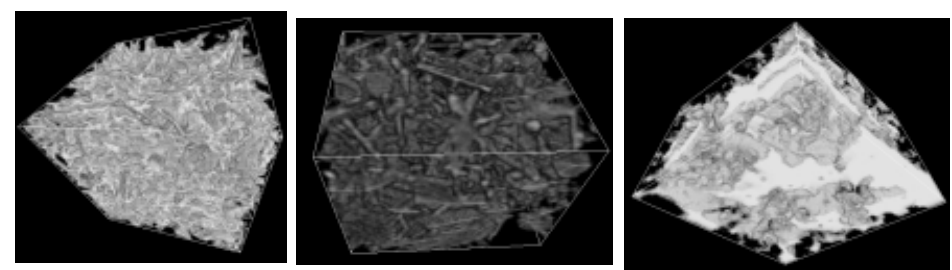

Fig. 4. Three dimensional renderings of individual classes (class 0 is on the left, class1 is in the middle, and class 2 is on the right) at 4 hours of hydration.

\subsection{Genetic Programming}

Genetic Progamming [20] 21][22] is a technique that derives a sequence of operations (chosen from a user defined set) that have high fitness (as defined by the user) using techniques inspired by evolution. We use GPP (Genetic Program-

\footnotetext{
${ }^{1}$ The identification of any commercial product or trade name does not imply either endorsement or recommendation by the National Institute of Standards and Technology.
} 

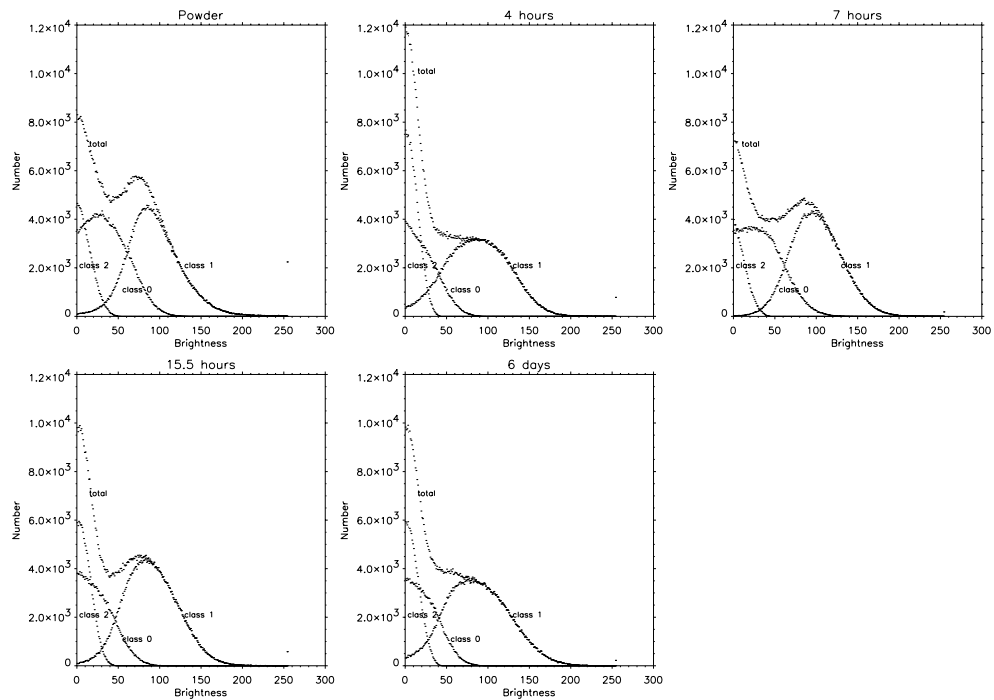

Fig. 5. Brightness histograms for whole datasets as well as component classes for powder, 4 hours, 7 hours, 15.5 hours, and 6 days.

ming - Procedural) [23] 24], a procedural genetic programming system that we have developed.

GPP was used to derive simple, understandable formulae that closely match the original classifications provided by autoclass. Before GPP is used, the class $1 / 2$ decision algorithm is determined by C5 as described above. GPP is then used to derive the class $0 / 1$ and the class $0 / 2$ decision algorithms. The method for using GPP in this problem followed the following steps for each desired classification:

- Prepare training data sets from the classified data sets.

- Select parameters, such as the operator set, for the GPP runs.

- Construct a fitness function to measure algorithm effectiveness.

- Execute a set of GPP runs.

- Select the run with results that most closely match the original classification.

- Simplify the GPP-produced algorithm to a succinct form.

All GPP runs were done with the same set of operating parameters. We used a small set of arithmetic and logical operators, a population size of 500, and the maximum number of generations for a single evolution was 50 .

The fitness function that we used is based on the correlation between the algorithm's classifications with the actual classifications. The fact that this is a two-valued problem simpifies the calculation of the correlation. We use the formulation by Matthews[25], which has been used in the context of genetic programming by Koza[21]. The correlation is given by: 


$$
\frac{T_{p} T_{n}-F_{n} F_{p}}{\sqrt{\left(T_{n}+F_{n}\right)\left(T_{n}+F_{p}\right)\left(T_{p}+F_{n}\right)\left(T_{p}+F_{p}\right)}}
$$

where:

$-T_{p}$ is the number of true positives

$-T_{n}$ is the number of true negatives

$-F_{p}$ is the number of false positives

$-F_{n}$ is the number of false negatives

The correlation is evaluated based on the execution of an algorithm on the appropriate training set. This correlation value varies from -1 to 1 , where 1 indicates perfect performance on the training set and -1 indicated a perfectly incorrect performance on the training set. Because these decision algorithms are binary in nature, we can turn a very bad algorithm into a very good algorithm simply by inverting each decision. In terms of the correlation value, this means that we can regard a decision algorithm with a correlation of -0.6 to be just as good as an algorithm with correlation +0.6 . So our fitness function is the absolute value of the correlation value given above. Each GPP run seeks to evolve an algorithm that maximizes this fitness value.

For each decision algorithm to be derived, five hundred GPP runs were made. Each run differed only in the seed to the random number generator. Given the resources available to us, we easily ran all 12 sets of runs over a single night.

After completing a set of five hundred runs we evaluated the algorithm generated by each run on the full data sets. This enables us to select the best performing algorithm. We then use a symbolic computation system (Maple [26]) to reduce the algorithms to simpler forms.

\section{Results}

Relatively simple decision rules were derived for all five time steps and for each of the required classifications. After deriving the rules, we sought to evaluate their effectiveness relative to the original autoclass classification.

\subsection{Rules}

Here are the classification algorithms that were derived by C5 and GPP. Note that the derived rules are succinct and the entire classification algorithm for a particular time step is quite compact.

\section{Powder}

if $A 1 \leq 42$.

then (class is either 2 or 0 )

$$
\text { if }\lfloor .518494 A 3+.019318 A 6\rfloor=2
$$




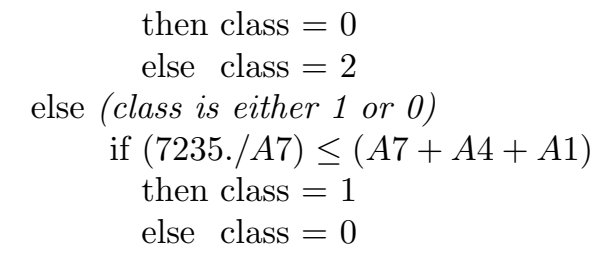

\section{4 hours}

if $A 1 \leq 27$.

then (class is either 2 or 0 )

if $A 6=0$

then if $\lfloor 0.5+.06145 A 1\rfloor=2$

then class $=0$

else class $=2$

then if $\lfloor 0.5+.06145 A 1+.003373 A 3\rfloor=2$

then class $=0$

else class $=2$

else (class is either 1 or 0 )

if $(6323.0 / A 1)<(A 0+A 4+0.692129 A 6+A 7)$

then class $=1$

else class $=0$

\section{7 hours}

if $A 1 \leq 42.5$

then (class is either 2 or 0 )

if $\lfloor .527467 A 1+.027467 A 7\rfloor=2$

then class $=0$

else class $=2$

else (class is either 1 or 0 )

if $\lfloor 0.5+0.008515 A 7\rfloor=1$

then class $=1$

else class $=0$

\section{5 hours}

if $A 1 \leq 30$.

then (class is either 2 or 0 )

if $\lfloor .525849(A 6+A 3)\rfloor=2$

then class $=0$

else class $=2$

else (class is either 1 or 0 )

if $A 0 \neq 0$

then if $A 4<(5321.0 / A 7)-(a 0+a 7)$

then class $=0$

else class $=1$

else if $A 4<(5321.0 / A 7)-a 3$ 


$$
\begin{aligned}
& \text { then class }=0 \\
& \text { else } \text { class }=1
\end{aligned}
$$

\section{6 days}

$$
\begin{aligned}
& \text { if } A 1 \leq 28 . \\
& \text { then class }=2 \\
& \text { else }(\text { class is either } 1 \text { or } 0) \\
& \text { if } A 0+(A 1-(4643 / A 7))>0 \\
& \text { then class }=0 \\
& \text { else class }=1
\end{aligned}
$$

\subsection{Evaluation of the Rules}

We use sensitivity and positive predictive value [27] as metrics to evaluate our rules. A rule can be optimal with respect to a particular classification in two ways. The rule can be very successful at seeing a class when it is there. This is called its sensitivity. And the rule can be very successful at identifying the class in the presence of other classes. This is called its positive predictive value. Let $T_{p}$ be the true positives. Let $F_{p}$ be the false positives. Let $F_{n}$ be the false negatives. Then:

$$
\begin{aligned}
\text { Sensitivity } & =\frac{T_{p}}{T_{p}+F_{n}} \\
\text { Positive-Predictive-Value } & =\frac{T_{p}}{T_{p}+F_{p}}
\end{aligned}
$$

In a confusion matrix, sensitivity is accuracy across a row; positive predictive value is accuracy down a column.

We test our classification rules with a completely different $100^{3}$ subcube of data from each of the five time periods. To test the rules we first compute the same attribute vectors for the new dataset. Then we use the prediction capability of autoclass to label the vectors. Finally, we use the above rules to create confusion matrices of the preditions for each of the time periods. We derive the sensitivity and positive predictive values for class and time period. The derived rules are all highly predictive as shown in the following table.

\begin{tabular}{|r|c|c|c|c|c|c|}
\hline \hline Dataset & \multicolumn{3}{|c|}{ Sensitivity } & \multicolumn{3}{c|}{ Positive-Predictive-Value } \\
\hline & class 0 & class 1 & class 2 & class 0 & class 1 & class 2 \\
Powder & 0.94 & 0.88 & 0.97 & 0.86 & 0.97 & 0.95 \\
4 Hour & 0.97 & 0.96 & 0.97 & 0.93 & 0.98 & 0.997 \\
7 Hour & 0.95 & 0.95 & 0.99 & 0.95 & 0.95 & 0.99 \\
15.5 Hour & 0.96 & 0.93 & 0.98 & 0.93 & 0.96 & 0.996 \\
6 Day & 0.97 & 0.96 & 0.996 & 0.99 & 0.95 & 0.98 \\
\hline \hline
\end{tabular}

\section{Conclusions and Future Work}

We have taken unclassified data and created a set of simple rules that accurately predict the class of unseen data for plaster powder, and plaster after 4, 7, 15.5 
hours, and 6 days of hydration. This was accomplished using a combination of three machine learning methods, providing results and insight that were not possible with any one of the techniques.

Our work on plaster has just begun, however. First, we would like to develop a better method for validating the classifications. One approach is to generate simulated plaster data sets for which proper classifications are known, for example using computer model microstructures designed to mimic the Plaster of Paris system [28]. We will also be working with an expert to label manually small subsets of the X-ray tomography data sets. These labeled data will then be used for training and validation. This will likely result in refinement of our rules.

Next we would like to develop equations that accurately predict the class regardless of the time of hydration, i.e. that work over the whole hydration period. We will need additional data to include variations with respect to the parameters that can influence the setting process and the resultant properties of plaster. Finally, we would like to predict physical characteristics of classes with equations, instead of predicting classes.

Plaster is an interesting and exciting topic for automated discovery methods. We look forward to extending our study.

Acknowledgements. We would like to thank Dale Bentz for his encouragement and support.

\section{References}

1. Kingrey, W.D., Bowen, H.K., Uhlmann, D.R.: Introduction to Ceramics. John Wiley and Sons, New York (1976)

2. Bullard, J.W.: Personal communication (2002)

3. Clifton, J.R.: Some aspects of the setting and hardening of gypsum plaster. Technical Note 755, NBS (1973)

4. Hand, R.J.: The kinetics of hydration of calcium sulphate hemihydrate: A critical comparison of the models in the literature. Cement and Concrete Research $\mathbf{2 4}$ (1994) 885-895

5. Ridge, M.J.: A discussion of the paper: The kinetics of hydration of calcium sulphate hemihydrate: A critical comparison of the models in the literature by $r$. j. hand. Cement and Concrete Research 25 (1995) 224

6. Hand, R.J.: A reply to a discussion by $\mathrm{m}$. j. ridge of the paper: The kinetics of hydration of calcium sulphate hemihydrate: A critical comparison of the models in the literature. Cement and Concrete Research 25 (1995) 225-226

7. D. P. Bentz, S. Mizell, S. G. Satterfield, J. E. Devaney, W. L. George, P. M. Ketcham, J. Graham, J. Porterfield, D. Quenard, F. Vallee, H. Sallee, E. Boller, J. Baruchel: The Visible Cement Dataset. J. Res. Natl. Inst. Stand. Technol. 107 (2002) 137-148

8. The visible cement dataset (2002) [online] <http://visiblecement.nist.gov> .

9. Cheeseman, R., Kelley, J., Self, M., Taylor, W., Freeman, D.: Autoclass: A bayesian classification system. In: Proceedings of the Fifth International Conference on Machine Learning, San Francisco, CA, Morgan Kaufman (1988) 65-74 
10. Cheeseman, P.: On finding the most probable model. In Shrager, J., Langley, P., eds.: Computational Models of Discovery and Theory Formation. Morgan Kaufman, San Francisco, CA (1991) 73-96

11. Stutz, J., Cheeseman, P.: Bayesian classification (autoclass): Theory and results. In Fayyad, U.M., Piatetsky-Shapiro, G., Smyth, P., Uthurusamy, R., eds.: Advances in Knowledge Discovery and Data Mining. AAAI Press, Menlo Park, CA (1995)

12. Kanefsky, B., Stutz, J., Cheeseman, P., Taylor, W., Clifton, J.R.: An improved automatic classification of a landsat/tm image from kansas (fife). Technical Report FIA-94-01, NASA AMES (1994)

13. Goebel, J., Volk, K., Walker, H., Gerbault, P., Cheeseman, P., Self, M., Stutc, J., Taylor, W.: A bayesian classifiection of the iras lrs atlas. Astronomy and Astrophysics 222 (1989) L5-L8

14. Reichenbach, H.: Atom and Cosmos. Dover Publications, Inc., Mineola, New York (1932) (First published in 1930 as Atom und Kosmos.).

15. Bentz, D.P.: Personal communication (2002)

16. Sattler, H., Bruckner, H.P.: Changes in volume and density during the hydration of gypsum binders as a function of the quantity of water available. ZKG International $54(2001) 522$

17. : C5 (2002) [online] <http://www.rulequest.com> .

18. Quinlan, J.R.: C4.5: Programs for Machine Learning. Morgan Klauffann, San Mateo (1993)

19. Crow, E.L., Siddiqui, M.N.: Robust estimation of location. Journal of the American Statistical Associatiuon 63 (1967) 363-389

20. Koza, J.R.: Genetic Programming: On the Programming of Computers by Means of Natural Selection. MIT Press (1992)

21. Koza, J.R.: Genetic Programming II: Automatic Discovery of Reusable Programs. MIT Press (1994)

22. Koza, J.R., Andre, D., Bennett III, F.H., Keane, M.: Genetic Programming 3: Darwinian Invention and Problem Solving. Morgan Kaufman (1999)

23. Hagedorn, J., Devaney, J.: A genetic programming system with a procedural program representation. In: 2001 Genetic and Evolutionary Computation Conference Late Breaking Papers. (2001) 152-159 http://math.nist.gov/mcsd/savg/papers ${ }^{\sim}$.

24. Devaney, J., Hagedorn, J., Nicolas, O., Garg, G., Samson, A., Michel, M.: A genetic programming ecosystem. In: Proceedings 15th International Parallel and Distributed Processing Symposium, IEEE Computer Society (2001) 131 http://math.nist.gov/mcsd/savg/papers .

25. Matthews, B.W.: Comparison of the predicted and observed secondary structure of t4 phage lysozyme. Biochimica et Biophysica Acta 405 (1975) 442-451

26. Monagan, M.B., Geddes, K.O., Heal, K.M., Labahn, G., Vorkoetter, S.M., McCarron, J.: Maple 6 Programming Guide. Waterloo Maple Inc., Waterloo, Ontario, Canada (2000)

27. Lathrop, R., Erbdster, T., Smith, R., Winston, P., Smith, T.: Integrating ai with sequence analysis. In Hunter, L., ed.: Aritifical Intelligence and Molecular Biology, Cambridge, MA (1993)

28. Meille, S., Garboczi, E.J.: Linear elastic properties of 2-d and 3-d modles of porous materials made from elongated objects. Mod. Sim. Mater. Sci 9 (2001) 1-20 